\title{
Ontology and Quantum Mechanics
}

\author{
N. D. Hari Dass
}

Tata Institute of Fundamental Research (TIFR), TIFR Centre for Interdisciplinary Sciences (TCIS), Hyderabad, India

Chennai Mathematical Institute, Chennai, India

Centre for Quantum Information and Quantum Computation, Indian Institute of Science, Bangalore, India

E-mails:dass@cts.iisc.ernet.in,dass@cmi.ac.in

Editors: Danko Georgiev \& Tabish Qureshi

Article history: Submitted on June 17, 2014; Accepted on August 3, 2014; Published on September 23, 2014.



he issue of ontology in quantum mechanics, or equivalently the issue of the reality of the wave function is critically examined within standard quantum theory. It is argued that though no strict ontology is possible within quantum theory, ingenious measurement schemes may still make the notion of a FAPP ontology (ontology for all practical purposes) meaningful and useful.

Quanta 2014; 3: 47-66.

\section{Introduction}

A cursory check as to the meaning of the word Ontology will turn up a bewildering response, with a wide spectrum of interpretations. So is also the case for its close relative Epistemology. It is not the purpose of this article to get into a general discourse on this concept. Instead, it will focus on its meaning as widely understood by physicists, more particularly the quantum physicists. Though notions of existence and of reality come frequently associated with ontology, we shall focus more on aspects of reality. In the specific context of quantum theory, this more or less concerns the so called reality of the wavefunction. Reality is in itself a heavily loaded concept were one to turn into

(c) (1) This is an open access article distributed under the terms of the Creative Commons Attribution License CC-BY-3.0, which permits unrestricted use, distribution, and reproduction in any medium, provided the original author and source are credited. it from general philosophical considerations. We shall therefore restrict attention to Physicist's notion of reality, however unsophisticated it may appear to philosophers at large!

It is fair to say that the notion of reality to most physicists is conditioned by their experience from classical physics. Many so called paradoxes in quantum theory have in fact arisen because of this. Nevertheless, a careful examination of the concept of reality in classical physics is essential as a guide to examining its counterpart in quantum theory. It is clear that even in classical physics, notions of reality are intimately tied up with aspects of observation, or of measurements. Therefore, the plan of this article is to first examine ontology in classical physics, and to identify those aspects of classical measurements and dynamics that make the notion of reality reliable and useful. We then examine the issue of ontology in quantum mechanics against the backdrop of a variety of quantum measurements all the way from the Dirac-von Neumann description to the current day explosions.

\section{Ontology in Classical Physics}

Reality in classical physics may be characterized by certain robust associations of attributes and objects. For example, when one says that a particular Rose is Red, this represents an element of reality with many important aspects, many of which appear trivial and straightforward unless carefully contemplated upon. In this case the attribute is Redness and the object is the Rose in question. 
What are the mechanisms in classical physics that bring about this association, and in what sense this association is robust are questions whose answers hold the key to a finer understanding of reality in classical physics.

Before attempting to answer them, let us expand the list of attributes in this case to include, let us say, Smell. Classical reality says that these two attributes can peacefully coexist and that the reality of one need not interfere with the reality of the other. Now what gives an element of reality to, say, the redness, is that no matter how many times we observe the color, no matter how we observe the color, or no matter how often we interject color observations with other observations, say in this case smell, we come up with the same measure of redness for the flower. It is obvious that this is possible only if observing the color of the rose does not itself alter its color.

We can sharpen this by introducing the notion of a state of the object. In the above example, red is the 'value' of the attribute of color for this particular state of the rose which may be called a 'red rose'. One could have yellow roses, purple roses etc. and they would all refer to different states of the object; let us stipulate that the state of any object is specified by the values of its attributes. It is worth recalling a characterization of a state by Dirac [1,2]; though it was given in the context of quantum theory, it is pertinent to any theory, and certainly to classical physics also. According to him, a state is an embodiment of all possible measurement outcomes.

At this point there is an important subtlety that needs to be taken care of. It appears and in reality it is indeed so, that a red rose is a different object from a yellow rose and we are not really talking of different states of an object but of different objects. In fact that may make the distinction between the object and its state artificial and unwarranted. To overcome that, we shall allow for the possibility (not altogether unrealistic) of processes that could change the color of a given rose. Then the rose, the given object, can indeed be in different states of color. If, as mentioned above, we are also considering additional attributes like smell, a characterization of the state of a rose would, in this classical context, require specifying the values of both smell and color. These values can be thought of as the outcomes of color and smell measurements thus tying up with the characterization of a state according to Dirac.

In fact, we can go to the more prosaic world of classical mechanics and consider a particle as the object, its position, velocity etc. as its attributes. The state of the particle is then specified by the values of these attributes. There is an obvious redundancy with this description. For example, one could have also considered the square of the position as an attribute, but then that would not carry any additional information from that already carried by the position on its own. So a distinction should be made be- tween what one may call primary and derived attributes. The upshot is that it is enough to consider an optimal set of independent attributes for state description.

Let us return to the issue of reality and its robustness. The association of, say, position with the particle can be taken as an element of reality which is robust because measurement of position of the particle returns the same values within some range of errors (more on this later) no matter how often this measurement is done, how this measurement is done (as there are many means of position measurements), and in what order these measurements are done in the sense that position measurements may be interspersed with measurements of other attributes. In fact, in the classical world it would then be possible to say that this element of reality exists even if no one is actually observing the particle!

It is obvious that this is possible if and only if the measurements have no effect on the state. Such measurements can be called non-invasive. But not every measurement should be necessarily non-invasive even in classical physics. One could in principle adopt a measurement scheme that is deliberately invasive. For example, a position measurement of a tiny particle could be done by hitting it with a big stone. So a choice of non-invasive measurement is essential in the scheme above. In the classical world, by and large, measurements are non-invasive unless by deliberate design.

It is important to emphasize that non-invasiveness by itself is enough to guarantee a robust element of reality. Now the other crucial aspect of the classical world enters the picture and this is determinism. To appreciate this consider the possibility that before a measurement the particle is in a definite state (state with definite values of all its attributes). A non-invasive measurement may leave the particle in the same state, but may not necessarily yield definitive values for these attributes. This could happen when the physical processes making up the measurement are not deterministic. It could well be that a definite measured value emerges upon averaging a large number of outcomes. Such a world would exhibit both ontic and epistemic features.

But the world of classical physics is deterministic. On top of that, no separate laws have to be stipulated for measurements. Therefore in principle every classical measurement should yield definite outcomes with no errors at all. But errors do occur in classical measurements. This is for the obvious reason that even in the deterministic world of classical physics, not every source of influence in an experiment can be identified and accurately accounted for. A pragmatic approach would treat the unknowns probabilistically thereby introducing randomness in a perfectly deterministic world! Therefore the outcomes will have variances and actual errors can be statistically reduced 
through repeated measurements. Nevertheless, even this randomness introduced purely for practical purposes, governs errors that can be controlled by better experimental designs. Then, one can adopt the reasonable stand that the outcomes within such narrow and controllable errors are, for all practical purposes, making the strictly non-invasive measurements into practically non-invasive measurements.

But it is worth appreciating that any randomness, however small, does not allow for ontic descriptions, in principle. But in practice this does not pose a problem. In that sense, even the 'real' world of classical physics has a blurry edge, which we ignore all the time!

All these considerations have one profound consequence. Measurements on a single object are meaningful, and statistical errors can be meaningfully reduced arbitrarily by making sufficiently large number of repeated measurements on the same object. It should be stressed that this arose both due to the near non-invasive measurements as well as due to each measurement practically yielding full information.

Even with regard to deliberately invasive measurements, the determinism of classical physics can in principle, though tedious and heavy on resources in practice, provide a means of compensating for the invasive effects. In the example of throwing a rock to measure the position of a small particle, though the rock strongly alters the state of the particle, very careful measurements of the subsequent trajectories of both the particle and the rock can be used to accurately reconstruct the state of the particle before the collision, and restore the particle to that state. But second law can put a limit on how much invasiveness is tolerable! If for example, the invasive measurement involved setting fire and vaporizing the particle, it would be practically impossible to regain the original state!

It is of course possible that the attributes change with time. The rose of the beginning could fade. Does this mean that the element of reality that was so carefully constructed was not real at all? The physicist's answer to this is not to deny the element of reality or its robustness, but to allow for a time evolution of states and their associated attributes. This is the idea of Dynamics. The determinism referred to earlier then takes the form of a Deterministic Dynamics. These deterministic rules of dynamics not only ensure unambiguous outcomes in ideal measurements, but they also ensure that no separate rules are necessary to describe measurements, unlike in quantum theory.

\section{Ontology in Quantum Mechanics}

At least as per our present understanding, the standard quantum theory is inherently random. From our previous discussion, no strict ontology ought to be possible then. Quantum Measurements, as understood during the critical years of the development of quantum theory, and as idealized by the Dirac-von Neumann measurement models, are certainly invasive, and uncontrollably so. They are invasive in an unpredictable way. This too leaves no room for an ontic description. The Born probability rule has to be invoked for a consistent interpretation of quantum mechanics and that is where randomness becomes intrinsic. Paradoxically, the rules for time evolution of states, or, quantum dynamics, is completely deterministic by itself! It is only measurement that brings in indeterminacy. Nevertheless, as we shall see later, there are intriguing pointers to why there can be no ontic description of quantum mechanics, coming from purely dynamical considerations (all unitary processes are considered dynamical here).

This also makes repeated measurements of the Diracvon Neumann meaningless when performed on a single copy. The simple reason being that the state after the first measurement bears no obvious relation to the state one started with, and the subsequent measurements can at best reveal the state after the first measurement. Therefore only ensemble measurements become significant. For a good account of the issues involved in getting information out of measurements on a single copy see [3].

If there are such serious obstacles to ontology in quantum mechanics, why bother to go further? There are several good reasons for it! Firstly, the extreme invasiveness of quantum measurements is certainly a distinctive feature of the Dirac-von Neumann, or more precisely, Projective Measurements. So the question naturally arises whether there can be other measurement schemes that are noninvasive or controllably non-invasive. It then becomes important to re-examine the ontology issue in the context of these alternate measurement schemes. As it turns out, there are so many interesting alternatives to projective measurements today [4]. It is the purpose of this article to do that examination carefully.

We set the following technical criterion for ontology: ontology is the ability to completely determine the previously unknown state of a single copy. Even in cases where this is not possible, we introduce the notion of FAPP Ontology (ontology For All Practical Purposes) as the ability to almost determine the unknown state of a single copy, or in other words, a state determination with specified amount of errors.

It may, however, be worthwhile to point out that strictly speaking, the condition of non-invasiveness may be too restrictive for ontology. Both in classical mechanics and 
in quantum mechanics if means are available for a complete determination of the state of a system albeit at the cost of a radical change of state (but not of the system itself!), one can still meaningfully ascribe reality to that state. Then one can prepare the system in this state, and as far as subsequent measurements are concerned, it would be as if the original state had not been tampered with. In the context of quantum mechanics, this entails simultaneous and accurate determination of the expectation values of non-commuting observables in a single measurement performed on a single copy. The uncertainty principle restricts the possible accuracies in such a measurement which resembles the well-known Arthurs-Kelly Measurements. Again, strict ontology would not be possible, but it would be interesting to investigate the best possible FAPP ontology.

Though historically it was not recognized as such, we can now trace all the essential non-classical features of quantum theory to just one principle, namely, The Principle of Superposition of States [1, 2, 5]. In fact, one can take this principle to be the defining feature of quantum theories. Other aspects like Entanglement, taken by many (particularly among the Quantum Information community) to be the crux of quantum mechanics, is a natural consequence of the superposition principle.

It turns out that even without a very detailed analysis, one can show the impossibility of perfectly non-invasive measurements in quantum mechanics by just invoking the superposition principle. We outline this powerful argument in subsection 3.1. Another, equally powerful argument against ontology can be given by invoking the No Cloning Theorem. The proof of the No Cloning Theorem involves only Unitarity, and makes no reference to quantum measurements at all. It is surprising that this theorem, which has nothing to do with the measurement process, could have such a strong bearing on the issue on ontology in quantum mechanics. This second argument is presented in subsection 3.2. We then analyse the projective measurements (section 4), the protective measurements section 5, a method of cloning which we had named Information Cloning (section 7), the weak measurements section 6, methods of approximate cloning section 8 for their implications on the question of ontology in quantum mechanics.

\subsection{Superposition Principle and Ontology}

Let us consider a hypothetical measurement device that is perfectly non-invasive (leaves the system state undisturbed). We can consider the initial unknown system state to be $|\psi\rangle_{S}$. Since this does not change, we can use a statevector representation for the system. The treatment of the apparatus will be more subtle. All that the apparatus is required to do is produce a probability distribution of outcomes which carries complete information about the expectation value of the observable in the system state $|\psi\rangle_{S}$. Therefore, at least the final state of the apparatus ought to be described by a density matrix. Then one might as well describe the entire history of the apparatus by a density matrix. Because the system stays in the same state throughout, it is consistent to treat the system by a state vector, and the apparatus by a density matrix. The initial state of the system-apparatus composite can be taken to be

$$
|\psi\rangle_{S} \otimes \rho^{A}(0)
$$

Under the measurement $\mathcal{M}$, this goes to

$$
|\psi\rangle_{S} \otimes \rho^{A}(0) \stackrel{\mathcal{M}}{\longrightarrow}|\psi\rangle_{S} \otimes \rho^{A}\left(\langle\psi|O| \psi\rangle_{S}\right)
$$

The measurement $\mathcal{M}$ not being a Unitary process, can take a pure density matrix to a mixed one. The final apparatus (reduced) density matrix is in general mixed. If such a $\mathcal{M}$ could be realized, it can be used as often as necessary to measure all the relevant observables for state tomography of $|\psi\rangle_{S}$ as the state is left undisturbed.

The map in Equation 2 is not consistent with the principle of linear superpositions of states. That is, if the measurement device works on $\left|\psi_{1}\right\rangle_{S}$ and $\left|\psi_{2}\right\rangle_{S}$, it will not work on an arbitrary superposition $\alpha\left|\psi_{1}\right\rangle+\beta\left|\psi_{2}\right\rangle$ i.e. the measurement does not work on an arbitrary unknown state. As this is a very important issue, let us state it as precisely as possible. For that, let us describe the system state also by a density matrix $\rho_{S}=|\psi\rangle\left\langle\left.\psi\right|_{S}\right.$ and recast Equation 2 as

$$
\rho_{S A}^{\mathrm{ini}}=\rho_{S} \otimes \rho^{A}(0) \stackrel{\mathcal{M}}{\longrightarrow} \rho_{S} \otimes \rho^{A}\left(\langle O\rangle_{\rho_{S}}\right)
$$

The map $\mathcal{M}$ can generically be expressed as

$$
\rho_{S A} \stackrel{\mathcal{M}}{\longrightarrow} \sum_{i} M_{i} \rho_{S A} M_{i}^{\dagger}
$$

The measurement operations $M_{i}$, in the sense of positiveoperator valued measures (POVMs), are independent of $\rho_{S A}$. Therefore, the 1.h.s. of Equation 3 is bilinear in the system state $|\psi\rangle_{S}$. If the system state $|\psi\rangle_{S}=\sum_{i} c_{i}|i\rangle_{S}$ in some basis $|i\rangle_{S}$, the 1.h.s. is a quadratic form in $\operatorname{Re} c_{i}, \operatorname{Im} c_{i}$. But the r.h.s. of the same equation is certainly not bilinear in this sense. Thus the conflict with the superposition principle.

This is a very powerful conclusion showing that the principle of linear superposition of states alone is enough to rule out ontology in quantum mechanics and one need not invoke the deep, but confusing, chain of arguments invoked by the founders like Niels Bohr. An explicit realization of this line of thinking is afforded by the measurements discussed in section 5 and section 7, In the 
case of Protective Measurements, the scheme requires the unknown initial states to be non-degenerate eigenstates of a suitable Hamiltonian. A linear superposition of such states is no longer a state of the same type. In the case of Information Cloning, the scheme requires the unknown states to be Coherent States of a Harmonic Oscillator, and again, a superposition of such coherent states is not a coherent state!

\subsection{The No-cloning theorem}

The No-Cloning theorem [6,7] is one of the most striking of all results in quantum theory! Invoking nothing more than the inner-product preserving nature of unitary transformations or the superposition principle, it states that no unitary process can ever 'copy' unknown quantum states. In a lighter vein it is said that there are no quantum Xerox machines! We shall first describe the theorem, which is remarkably straightforward considering its profundity.

Consider an unknown state $|\psi\rangle_{S}$ of some quantum system and $N$ identical copies of another, but known, state $|0\rangle_{S}$ of the same system (it is not really necessary that they be of the same system, though). The latter are also called 'blanks' or 'ancillaries'. A unitary transformation $\mathcal{U}$ acting on the tensor product Hilbert space $\mathcal{H}^{N+1}$ is said to be a universal cloning transformation if it satisfies

$$
\mathcal{U}|\psi\rangle \otimes|0\rangle_{1} \otimes \ldots \otimes|0\rangle_{N}=|\psi\rangle \otimes|\psi\rangle_{1} \otimes \ldots \otimes|\psi\rangle_{N}
$$

for every $|\psi\rangle$. The No-cloning theorem is a proof that no such universal unitary transformation can exist. For a proof based only on unitarity of $\mathcal{U}$, consider a second state $|\chi\rangle$ so chosen that $|\langle\chi \mid \psi\rangle| \neq 0,1$. Then the effect of $\mathcal{U}$ on $|\chi\rangle$ has to be

$$
\mathcal{U}|\chi\rangle \otimes|0\rangle_{1} \otimes \ldots \otimes|0\rangle_{N}=|\chi\rangle \otimes|\chi\rangle_{1} \otimes \ldots \otimes|\chi\rangle_{N}
$$

Taking the inner product between these two equations and using unitarity of $\mathcal{U}$, one gets,

$$
\langle\chi \mid \psi\rangle=(\langle\chi \mid \psi\rangle)^{N+1}
$$

But this is possible only if $|\langle\chi \mid \psi\rangle|=0,1$ which contradicts the initial premise about $|\chi\rangle$ ! The same proof can also be viewed as a consequence of the superposition principle.

What is the relevance of the No-cloning theorem to our discussion of ontology? The point is, that $N$ can be made very very large, at least in principle, either in a single application of the universal cloner or in many cascaded applications of it. Then we can set aside one out of $N+1$ copies produced, and use the remaining $N$ copies for an ensemble state determination. The accuracy of the subsequent state determination can be improved with higher and higher $N$. One would still be left with one copy of the original unknown state even if the tomography with the $N$ copies is as invasive as can be.

Thus if an universal cloner existed, one would in effect be able to make a non-invasive measurement on a single copy of an unknown state and still be able to determine its state as accurately as one wishes. It is rather remarkable that this theorem which invokes only aspects of unitary evolutions, with no explicit reference to quantum measurements, nevertheless captures the very essence of quantum measures as per the Copenhagen Interpretation! This deep connection also borders on the mystic.

However, we shall introduce a novel Information Cloning which bypasses the no cloning theorem in a subtle way and is a way of getting information on a single copy, albeit with errors that cannot be reduced arbitrarily.

Before proceeding, we wish to highlight some other aspects of the No-cloning theorem. As expressed in Equation 5, the cloning transformation has made additional copies of the unknown state while preserving the original. But for the purposes of ontology, the preservation of the original is quite unnecessary as long as one ends up with sufficient number of copies.

A particularly striking example where the original is totally destroyed but one copy is left behind is in Quantum Teleportation! In fact in that case, the copy is created in a way that it is physically separated by distance from the original. Complete destruction of the original with exactly one copy produced also happens in swapping, but unlike teleportation a unitary transformation can accomplish that. Though quantum teleportation is not particularly useful in determining the unknown original state, it brings to the fore another aspect of reality, namely, transport of 'real' objects. In classical mechanics for sure, an object that does not have reality, or does not exist, cannot be transported. So it would be legitimate in that context to say that anything that can be transported is also real. In that sense, quantum teleportation would accord some reality to the wavefunction (state). Being a single state there can be no strict ontology though a FAPP ontology would be possible. Since the state is not determined, there is no epistemology either.

\section{Projective Measurements and Ontology}

Now we analyze why the Projective or Dirac-von Neumann measurements cannot yield any ontology. Even though the arguments are simple and straightforward, we recast them in the language of joint and conditional probabilities so we can use the same framework to address the issue of ontology in other contexts like weak measurements. 
In a strong or projective measurement, the state after the first measurement is changed randomly to one of the eigenstates of the observable being measured. The outcome of the apparatus is the corresponding eigenvalue. The fact that a given eigenstate-eigenvalue combination could have resulted from infinitely many unknown initial states makes their reconstruction impossible from the information available after a single such measurement. Such a reconstruction requires an ensemble measurements with optimally chosen observables.

If repeated strong measurements are performed on a single copy, the second and all subsequent measurements are eigenstate measurements where the eigenstate in question is the state after the first measurement. Therefore all subsequent measurements leave the system in this same eigenstate and all subsequent apparatus outcomes are exactly the same as the outcome of the first measurement. In other words, they do not generate any additional information required for the state reconstruction. The strong measurements are not only highly invasive, they do not generate any information for determining the state. These are the reasons, within standard quantum mechanics, for the impossibility of an ontological description.

Now let us recast these considerations in the language of conditional and joint probabilities of outcomes of repeated measurements on a single copy. Let the observable being measured is $S$, with the spectrum $s_{i},\left|s_{i}\right\rangle_{S}$. If the initial unknown state of the system is

$$
|\psi\rangle_{S}=\sum_{i} \alpha_{i}\left|s_{i}\right\rangle_{S}
$$

The probability distribution for the outcomes of the first measurement is given by

$$
P\left(p_{1}\right)=\sum_{i}\left|\alpha_{i}\right|^{2} \delta\left(p_{1}-s_{i}\right)
$$

This says that the first outcome is random with the above distribution. Let the outcome of the second measurement be $p_{2}$, and as explained above, it has to be the same as $p_{1}$ because it is an eigenstate measurement. Therefore, the probability distribution for $p_{2}$ is conditional on the outcome $p_{1}$. In other words, the conditional probability distribution $P\left(p_{2} \mid p_{1}\right)$ for the outcome $p_{2}$, conditional on the first outcome being $p_{1}$ is

$$
P\left(p_{2} \mid p_{1}\right)=\delta\left(p_{2}-p_{1}\right)
$$

The Joint Probability Distribution $P\left(p_{2}, p_{1}\right)$ for the outcomes of the first two of the repeated measurements is now given by

$$
\begin{aligned}
P\left(p_{2}, p_{1}\right) & =P\left(p_{2} \mid p_{1}\right) P\left(p_{1}\right) \\
& =\sum_{i}\left|\alpha_{i}\right|^{2} \delta\left(p_{2}-p_{1}\right) \delta\left(p_{1}-s_{i}\right) \\
& =\sum_{i}\left|\alpha_{i}\right|^{2} \delta\left(p_{2}-s_{i}\right) \delta\left(p_{1}-s_{i}\right)
\end{aligned}
$$

It is straightforward to generalize these to the outcomes of $N$ repeated measurements on a single copy:

$$
P\left(p_{N}, \ldots, p_{1}\right)=\sum_{i}\left|\alpha_{i}\right|^{2} \prod_{j=1}^{N} \delta\left(p_{j}-s_{i}\right)
$$

As usual, it is useful to introduce $\mathrm{y}_{N}$ to be the average of the first $N$ outcomes, and consider its probability distribution $P(y)$ that is

$$
\mathrm{y}_{N}=\frac{\sum_{i} p_{i}}{N}
$$

and

$$
P\left(\mathrm{y}_{N}\right)=\int \ldots \int \prod_{i} d p_{i} P(\{p\}) \delta\left(\mathrm{y}_{N}-\frac{\sum_{i} p_{i}}{N}\right)
$$

where the notation $\{p\}$ is used to indicate the values of the set of all $p$-variables. On using Equation 12 and Equation 14, it follows that

$$
P\left(\mathrm{y}_{N}\right)=\sum_{i}\left|\alpha_{i}\right|^{2} \delta\left(\mathrm{y}_{N}-s_{i}\right)
$$

The repeated measurements have not changed the nature of the distribution at all, and it remains the same as Equation 9! Though our simple reasoning had already told us this, the formalism of conditional and joint probabilities used above will prove to be useful in more complicated situations where there are no such simple reasoning available.

\subsection{Sharpening the ontology criterion}

The form of Equation 15, derived for Projective Measurements which are decidedly invasive and hence incapable of any ontological descriptions, suggests an even more precise technical criterion for ontology. For that, let us contrast Equation 15 with what one would expect in the case of ensemble measurements on the basis of the Central Limit Theorem:

$$
P\left(\mathrm{y}_{N}\right)=N e^{-\frac{N\left(\mathrm{y}_{N}-\mu\right)^{2}}{\Delta^{2}}}
$$

This suggests a way to sharpen the criterion for onticity in quantum mechanics, given verbally earlier, to the following precise mathematical criterion: exact ontology in quantum mechanics is the ability to find non-invasive measurement schemes such that the mean of the $N$ outcomes of repeated measurements on a single copy of a system in an unknown state takes the deterministic form

$$
P\left(\mathrm{y}_{N}\right)=\delta\left(\mathrm{y}_{N}-\mu\right), \quad \mu=\langle\psi|O| \psi\rangle
$$

Not surprisingly, there will be no candidates within quantum mechanics for this criterion. 
The next best possibility will be the FAPP-Ontology discussed earlier. The following two criteria provide precise characterizations of such. The first is that the statistics of outcomes of repeated measurements on a single copy will be very similar to that obtained from measurements on an ensemble. In particular, the distribution for the average $\mathrm{y}_{N}$ will be a single distribution as in Equation 16, and additionally $\mu=\langle\psi|S| \psi\rangle$. The figures of merit for the FAPP ontology are (i) how close $\mu$ actually is to the expectation value, and (ii) how small the error $\epsilon=\frac{\Delta}{\sqrt{N}}$ is.

The second criterion allows for the distribution $P\left(\mathrm{y}_{N}\right)$ to deviate from a single distribution but with very small deviations

$$
P\left(\mathrm{y}_{N}\right)=p_{0} e^{-\frac{\left(y_{N}-\langle S\rangle_{\psi}\right)^{2}}{\epsilon^{2}}}+\sum_{i} p_{i} e^{-\frac{\left(\mathrm{y}_{N}-\mu_{i}\right)^{2}}{\epsilon_{i}^{2}}}
$$

In this case, the average outcome of repeated measurements will be random, and ensemble measurements become a necessity; measurements on a single copy will not reveal any information about the unknown state. In the coming sections we shall discuss explicit realizations of these criteria.

\section{Protective Measurements and Ontology}

Aharonov, Anandan and Vaidman [8, 9] proposed a remarkable type of experiments which they called Protective Measurements. They gave an explicit realization for them and showed that for a restricted class of states, and in a certain ideal limit, one could get full information about single copies of such restricted class of states without affecting the state. From whatever we have said so far, such a proposal would realize exact ontology in the ideal limit. Closer examination, however, shows that even these remarkable category of measurements actually provide only FAPP ontology, as the ideal limit requires measurements lasting infinitely long. Now we elaborate on the details.

They consider states that are non-degenerate eigenstates of some unknown Hamiltonian. For this reason, the states are indeed unknown. Let us briefly review the standard projective measurements to see the differences and commonalities between projective and protective measurements. For every type of measurement it is necessary to characterize the measuring apparatus. Niels Bohr was of the opinion that this necessarily had to be classical, whereas Dirac and von Neumann found it desirable to take this also to be a quantum system. It is also important to consider the modern picture of the Dirac-von Neumann Scheme. According to this, the final act of the measurement (the one that breaks the so called infinite von Neumann regression) is environmental decoherence which accounts for the real life situation that there is a complex environment with which both the system and the apparatus are interacting. This, technically speaking, renders the final density matrix diagonal in an apparatus Hilbert space basis which defines the Pointer States for the apparatus. Let $R_{A}$ be the observable of the apparatus whose eigenstates are the pointer states. In the Dirac-von Neumann measurement theory formalism, one introduces an apparatus operator $Q_{A}$ that is canonically conjugate to $R_{A}$ that is $\left[R_{A}, Q_{A}\right]=\imath \hbar$.

For both types of measurements, the interaction between the 'apparatus' and the system is taken to be described by a Hamiltonian:

$$
H_{I}(t)=g(t) Q_{A} S, \quad \int g(t) d t=1
$$

Here $S$ is the system observable that is being measured and $Q_{A}$ the observable of the apparatus described above. The integral condition on $g(t)$ is a convenient normalization which can be taken without loss of generality. In addition to this interaction Hamiltonian, the time evolution of both the system and the apparatus are respectively governed by their own Hamiltonians $H_{A}$ and $H_{S}$, respectively.

The projective measurements correspond to an impulsive $g(t)$ that is $g(t)$ is non-zero only in a very small time interval $-\frac{\epsilon}{2}<t<\frac{\epsilon}{2}$. The time-evolution unitary transformation taking pre-measurement-interaction states to post-measurement-interaction states is given by

$$
U\left(\frac{\epsilon}{2},-\frac{\epsilon}{2}\right)=e^{-\frac{l}{\hbar} \int_{-\frac{\epsilon}{2}}^{\frac{\epsilon}{2}} H d t}
$$

Normally this unitary transformation is given by time ordered integral over the total Hamiltonian $\mathrm{H}(\mathrm{t})$ :

$$
H(t)=H_{A}+H_{S}+H_{I}
$$

In the limit of the measurement interaction being extremely impulsive that is $\epsilon \rightarrow 0$, the time ordered integral is well approximated by

$$
U=e^{-\frac{l}{\hbar} Q_{A} S}
$$

It should be noted that $H_{A}, H_{S}$ do not contribute in this impulsive limit (it is understood that these Hamiltonians are bounded). The combined state of the system and apparatus before measurement is taken to be the disentangled state

$$
\left|t_{<}\right\rangle=|v\rangle_{S}\left|\Phi\left(r_{0}\right)\right\rangle_{A}
$$

The initial apparatus state, in the Dirac-von Neumann scheme is taken to be an eigenstate of $R_{A}$ with an eigenvalue, say, $r_{0}$; this corresponds to the initial reading of 
the apparatus. To avoid technical difficulties arising out of the use of continuous variables, the initial apparatus state $\left|\Phi\left(r_{0}\right)\right\rangle$ is taken to be a wavepacket sharply centered around the value $r_{0}$ of $R_{A}$.

Here $|v\rangle$ is the unknown system state on which a measurement of the observable $S$ is performed. If $\left|s_{i}\right\rangle$ are the eigenstates of $S$ that is $S\left|s_{i}\right\rangle=s_{i}\left|s_{i}\right\rangle$, and $|v\rangle=\sum_{i} \alpha_{i}\left|s_{i}\right\rangle$ the post-measurement interaction state is given by

$$
\left|t_{>}\right\rangle=U\left|t_{<}\right\rangle=\sum_{i} \alpha_{i} e^{-\frac{l}{\hbar} s_{i} Q_{A}}\left|s_{i}\right\rangle\left|\Phi\left(r_{0}\right)\right\rangle
$$

As $Q_{A}$ is canonically conjugate to $R_{A}$, the exponential operator shifts the value of $R_{A}$ by $s_{i}$ and one gets the entangled state

$$
\left|t_{>}\right\rangle=\sum_{i} \alpha_{i}\left|s_{i}\right\rangle\left|\Phi\left(r_{0}+s_{i}\right)\right\rangle
$$

This explicitly manifests the one-one correspondence between the states $\left|s_{i}\right\rangle$ of the system, and the states $\left|\Phi\left(r_{0}+s_{i}\right)\right\rangle$ of the apparatus. But the state in Equation 25 is entangled and it hardly reflects the single outcomes expected of a good measurement! It is instructive to see how decoherence 'solves' this issue; for that, consider the pure density matrix corresponding to this state:

$$
\rho^{S+A}\left(t_{>}\right)=\sum_{i, j} \alpha_{i} \alpha_{j}^{*}\left|s_{i}\right\rangle\left\langle s_{j}|| \Phi\left(r_{0}+s_{i}\right)\right\rangle\left\langle\Phi\left(r_{0}+s_{j}\right)\right|
$$

Clearly this matrix is not diagonal in the pointer basis $|\Phi\rangle$. Decoherence reduces this to the mixed density matrix

$$
\rho^{S+A}\left(t_{>}\right)=\sum_{i}\left|\alpha_{i}\right|^{2}\left|s_{i}\right\rangle\left\langle s_{i}|| \Phi\left(r_{0}+s_{i}\right)\right\rangle\left\langle\Phi\left(r_{0}+s_{i}\right)\right|
$$

Though this still does not explain how single outcomes come about, it has at least reduced that to a classical problem of picking from a mixture, much like picking a card out of a deck.

To pictorially contrast the projective and protective cases, we show in Figure 1a the outcomes of a standard Stern-Gerlach experiment viewed as a projective measurement. With this background, it is easy to grasp the essentials of a Protective Measurement. The major departure from projective measurements is that now the measurement interaction behaves oppositely to what it did in the case of projective measurements-the interaction time $T$ is now taken to be very large, approaching infinity! Let us leave aside for now questions like the meaning of measurements that take infinitely long, and proceed. It is simplest to take $g(t)$ to be a constant. Then the normalization condition gives $g=\frac{1}{T}$, where $T$ is the long duration of the measurement, which will tend to $\infty$ in the end. The total Hamiltonian becomes

$$
H=H_{A}+H_{S}+\frac{1}{T} Q_{A} S
$$

which is time independent. Again, for simplicity we restrict analysis to the choice $\left[H_{A}, Q_{A}\right]=0$ (for a complete discussion of the general situation see [10]). However, even in the standard Stern-Gerlach case, such a simplification does not happen. This condition allows both $H_{A}, Q_{A}$ to be simultaneously diagonalized

$$
Q_{A}\left|a_{i}\right\rangle_{A}=a_{i}\left|a_{i}\right\rangle_{A}, \quad H_{A}\left|a_{i}\right\rangle_{A}=E_{i}^{A}\left|a_{i}\right\rangle_{A}
$$

$H_{S}$ taken to be unknown has the non-degenerate eigenstates $|j\rangle_{S}$, with eigenvalues $\omega_{j}$. Because of the simplifying assumptions made, the total Hamiltonian $H$ also commutes with $Q_{A}$ and both of them can also be diagonalized simultaneously. If we take $H_{A}\left|a_{i}\right\rangle_{A}=E_{i}^{A}\left|a_{i}\right\rangle_{A}$, the simultaneous eigenstates of $H$ and $Q_{A}$ are of the form $|j, i\rangle_{S}\left|a_{i}\right\rangle_{A}$ with $|j, i\rangle_{S}$ satisfying

$$
\left(H_{S}+\frac{1}{T} a_{i} S\right)|j, i\rangle_{S}=\Omega(j, i)|j, i\rangle_{S}
$$

It is clear that $\Omega(j, i) \stackrel{T \rightarrow \infty}{\longrightarrow} \omega_{j}$ and $|j, i\rangle_{S} \stackrel{T \rightarrow \infty}{\longrightarrow}|j\rangle_{S}$. The eigenvalues and eigenstates of the total Hamiltonian $H$ can now be expressed as

$$
\begin{aligned}
H|j, i\rangle_{S}\left|a_{i}\right\rangle_{A} & =E(j, i)|j, i\rangle_{S}\left|a_{i}\right\rangle_{A} \\
& =\left(E_{i}^{A}+\Omega(j, i)\right)|j, i\rangle_{S}\left|a_{i}\right\rangle_{A}
\end{aligned}
$$

For very large $\mathrm{T}, \Omega(j, i)$ can be calculated in first order perturbation theory to get

$$
\Omega(j, i)=\omega_{j}+\frac{1}{T} a_{i}\langle j|S| j\rangle_{S}
$$

If the unknown system state before measurement is the non-degenerate eigenstate $|k\rangle_{S}$ of $H_{S}$, the joint state before measurement is taken to be $|k\rangle_{S}\left|\Phi\left(r_{0}\right)\right\rangle_{A}$, with $\left|\Phi\left(r_{0}\right)\right\rangle_{A}$ being the same as what was used in projective measurements.

The joint state after time $T$ is

$$
\begin{aligned}
|k, T\rangle & =U(T)|k\rangle_{S}\left|\Phi\left(r_{0}\right)\right\rangle_{A} \\
& =\sum_{i, j}\left\langle a_{i} \mid \Phi\left(r_{0}\right)\right\rangle_{A}\langle j, i \mid k\rangle_{S} e^{-\frac{l}{\hbar} T E(j, i)}|j, i\rangle_{S}\left|a_{i}\right\rangle_{A}
\end{aligned}
$$

In first order perturbation theory, $\langle j, i \mid k\rangle_{s}=\delta_{j, k}$. Putting everything together

$$
|k, T\rangle \stackrel{T \rightarrow \infty}{\longrightarrow} e^{-\frac{l}{\hbar} \omega_{k} T}|k\rangle_{S} e^{-\frac{l}{\hbar} H_{A} T} e^{-\frac{l}{\hbar}\langle k|S| k\rangle_{S} Q_{A}}\left|\Phi\left(r_{0}\right)\right\rangle_{A}
$$

In other words

$$
|k, T\rangle \rightarrow e^{-\frac{l}{\hbar} \omega_{k} T}|+\rangle e^{-\frac{l}{\hbar} H_{A} T}\left|\Phi\left(r_{0}+\langle k|S| k\rangle\right)\right\rangle_{A}
$$

Thus under these protective measurements, the original state is protected and the apparatus reads the expectation value $\langle k|S| k\rangle_{S}$ ! This is modulo the $e^{-\frac{l}{\hbar} H_{A} T}$ factor. 


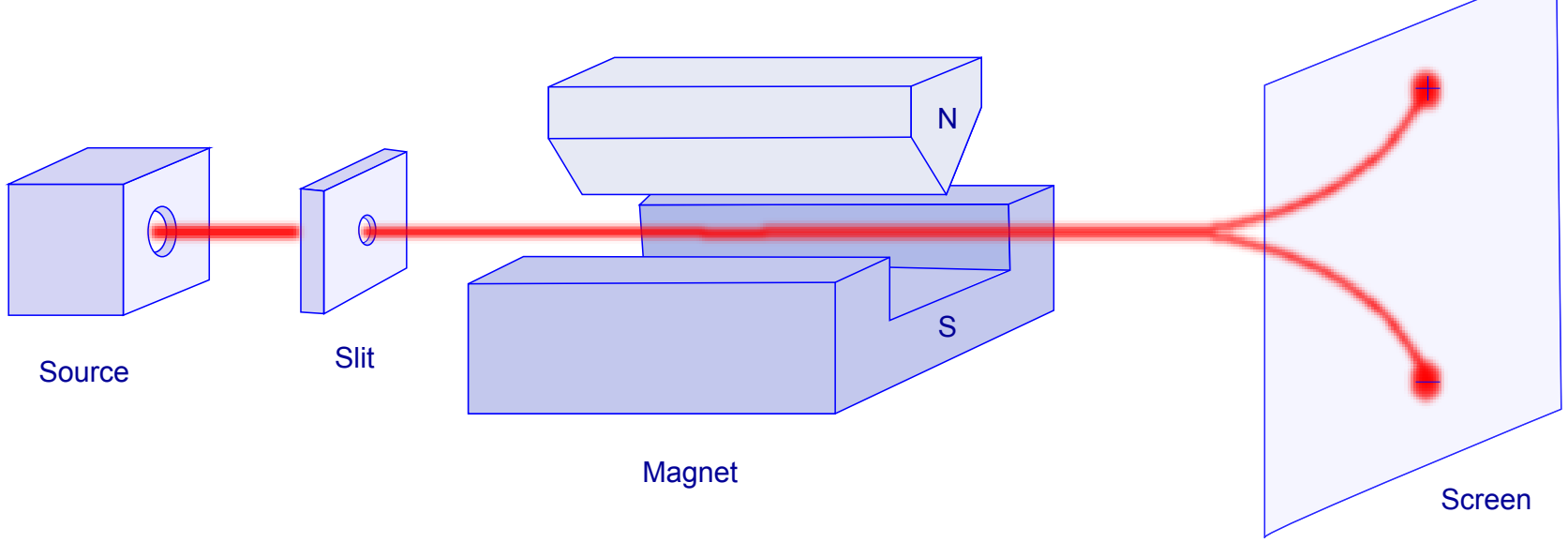

B

B

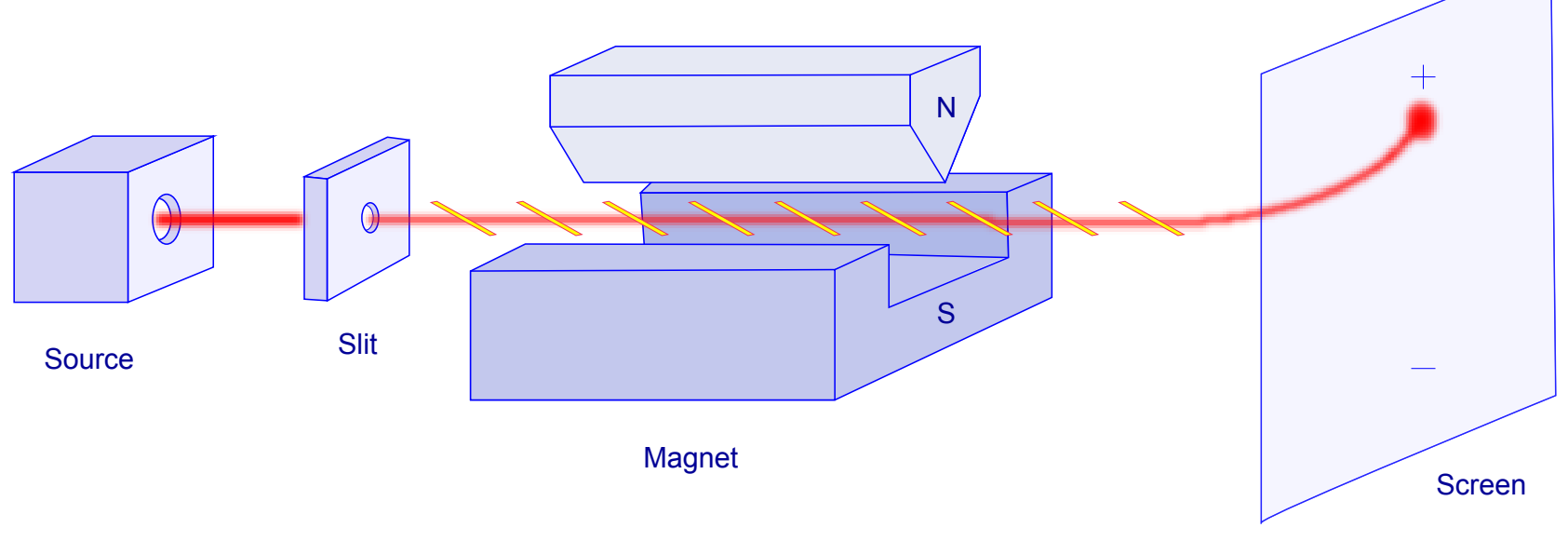

C

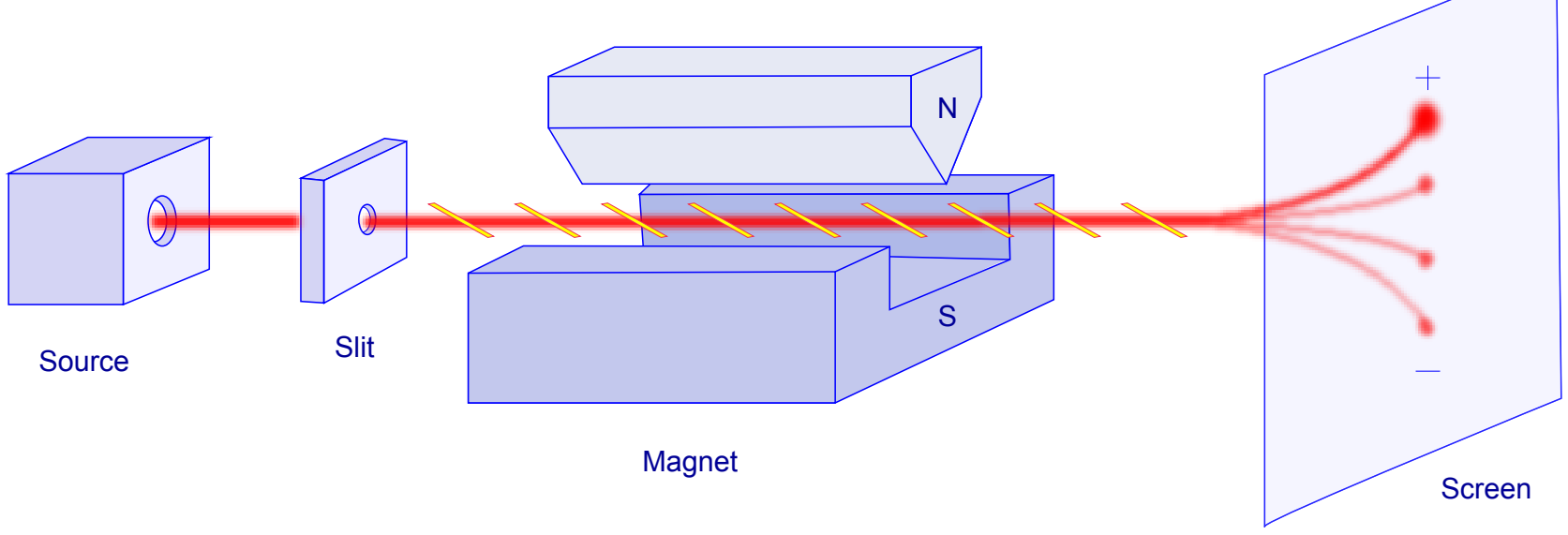

Figure 1: A. The standard Stern-Gerlach measurement setup denoting a projective measurement on a spin- $\frac{1}{2}$ system. The source emits a collimated beam of silver atoms directed between the poles of a magnet that produces an inhomogeneous magnetic field. Since the silver atoms are neutral they do not experience a Lorentz force. However, silver has in its outer shell an unpaired $5 \mathrm{~s}$ electron that is in a zero orbital angular momentum state. Thus the deflection by the inhomogeneous magnetic field is due to the spin of the outer electron only. The top (+) and the bottom (-) spots are hit randomly with probabilities given by the initial state. $B$. The situation to be expected in the case of an ideal protective measurement (that is in the extreme adiabatic limit $T \rightarrow \infty$ ). In this case, the beam hits the screen at only one spot which directly measures the expectation value of the observable in the initial state. The slashes denote the 'protective magnetic field' which has to be present over and above the inhomogeneous magnetic field. C. The situation in the case of the non-ideal protective measurement (that is when $T$ is very large but finite). In this case, while the spot occurring in the ideal case has a very large probability, other cases as enumerated in subsection 5.1 loccur with small but non-vanishing probabilities. 
Since the state is 'undisturbed', one can reuse it for carrying out protective measurements of all the necessary observables for complete state determination. The apparatus and the system are disentangled, and there is no need to take recourse to decoherence to achieve the final step in the measurement process. This is what can be called the Ideal protective measurements, in the sense that it is valid only in the strict $T=\infty$ limit. Figure 1b shows the situation to be expected for an ideal protective SternGerlach experiment. Unlike the standard Stern-Gerlach set up, the silver atoms in an ideal protective measurement would strike the screen at only one spot, in between the extreme positions encountered in the standard case. Its location is a precise measure of the expectation value of the measured observable in the unknown initial state.

But Equation 35 is precisely the kind that had been argued to be in conflict with the superposition principle in subsection 3.1! The Aharonov-Anandan-Vaidman scheme cleverly evades this by considering the unknown initial states to be non-degenerate eigenstates of $H_{S}$; therefore, superpositions of such states can no longer be nondegenerate eigenstates of $H_{S}$ !

\subsection{Non ideal protective measurements}

The Ideal case is obviously unphysical as it is meaningless for any measurement to last infinitely long! In real life situations $T$ can be very very large (compared to the time scales involved) but not $\infty$. One may naively argue that for all practical purposes the difference between such very large $\mathrm{T}$ and the ideal limit should be negligible. Indeed, for ensemble measurements the difference between very large $T$ and $T=\infty$ is negligible in the precise sense that the resulting probability distributions for outcomes differ only very slightly, and all the statistical conclusions are not affected significantly.

But for measurements on single copies, which are the only relevant measurements in the context of ontology, the situation is dramatically different. In Quantum Mechanics, unlike in the classical counterpart, individual outcomes of measurements are completely random and unpredictable. Even outcomes with hopelessly small probabilities can manifest. Only if their probability is exactly zero, will they not show up. This makes a very significant difference for protective measurements. In a nutshell, departure from $T=\infty$ causes a very small but significant entanglement between the system and the apparatus. This can cause the first protective measurement to project the unknown initial state into any state that is orthogonal to it. This way, not only is the state not protected during the first measurement, it renders meaningless the outcome of even protective measurements subsequently. No state reconstruction is possible and there is no strict ontology. This was the criticism of protective ontology that was made by both [10] as well as by [11].

To address these issues we need to consider all sources of $\frac{1}{T}$ corrections to the ideal results. We refer the reader to [10,12,13] for the technical details. Here we shall list the important sources of $\frac{1}{T}$ corrections and discuss their importance. In the sum of Equation 33, one will have to take into account system states $|j \neq k, i\rangle_{S}$. In order to get the leading $\frac{1}{T}$ corrections, second order perturbation theory becomes necessary. This typically introduces corrections of the type $\left|k^{\prime}\right\rangle_{S} Q_{A}^{2}\left|\Phi\left(r_{0}\right)\right\rangle$. Schematically the effect of these corrections can be represented as

$$
\left.|T\rangle=\mid \text { ideal }\rangle+\frac{c}{T} \mid \text { non-ideal }\right\rangle
$$

It is important to note that $\langle$ ideal|non-ideal $\rangle=0$ because of the nature of perturbation theory. Now we can further enumerate some possibilities:

- State is protected and apparatus reads the $\left\langle Q_{S}\right\rangle$ in that state with $P=1-\frac{c^{2}}{T^{2}}$.

- State protected but pointer in all possible states with probability $\simeq \frac{1}{T^{2}}$.

- State collapses to the state orthogonal to it and the pointer reads the expectation value in the orthogonal state with probability $\simeq \frac{1}{T^{2}}$.

- State collapses to the orthogonal but pointer in all possible states with probability $\simeq \frac{1}{T^{2}}$

This is depicted pictorially in Figure 1c. It is worth emphasizing that in each of these cases, the system state after measurements remains correlated with the original state. This is in sharp contrast to projective measurement where the system state after the measurement is completed has no memory of the original state whatsoever.

\subsection{Adiabatic two qubit interactions-another twist}

As a further generalization of the protective measurement schema, Anirban Das and myself [12] considered the case where the role of the apparatus is also played by another qubit or by a quantum system with finite dimensional Hilbert space. Let us illustrate with the example of the qubit as a detector. We take the basis states to be $\left|d_{\uparrow}\right\rangle_{A}$ and $\left|d_{\downarrow}\right\rangle_{A}$. The system is also taken to be a qubit with its Hilbert space spanned by $|\uparrow\rangle_{S}$ and $|\downarrow\rangle_{S}$. The measurement interaction is taken to be represented by the unitary transformation $\mathcal{U}$ :

$$
\begin{aligned}
& |\uparrow\rangle\left|d_{\downarrow}\right\rangle \stackrel{\mathcal{u}}{\rightarrow}|\uparrow\rangle\left|d_{\uparrow}\right\rangle \\
& |\downarrow\rangle\left|d_{\downarrow}\right\rangle \stackrel{\mathcal{u}}{\rightarrow}|\downarrow\rangle\left|d_{\downarrow}\right\rangle
\end{aligned}
$$


The components of spin are taken to be along the $z$-axis for both systems. For projective measurements where there are only two possible outcomes, it suffices to take $\left|d_{\uparrow}\right\rangle,\left|d_{\downarrow}\right\rangle$ as the pointer states. Whether there are any realistic 'environments' that can result in decoherence in this basis is not very clear. For adiabatic measurements where there can be a near-continuum of outcomes, we shall take angular momentum coherent states obtained by rotating, say, $\left|d_{\uparrow}\right\rangle$ by $\theta$ about the $x$-axis as the pointer states. Once again the existence of suitable decoherence mechanisms in this basis remains to be understood. More general possibilities for pointer states can also be considered. The interaction Hamiltonian that generates the unitary transformation $\mathcal{U}$ turns out to be (actually there are infinitely many such Hamiltonians!)

$$
-\pi g(t) P_{z,+}^{S} \otimes P_{x,-}^{A}
$$

Here $P_{a, \pm}$ are the projection operators for spin \pm along the $a$-direction. $H_{A}$ is taken to be the rotationally invariant $\vec{S}_{A} \cdot \vec{S}_{A}$. This, being a constant, does not lead to any pointer state broadening. Let the initial unknown system state be

$$
|v\rangle=\alpha|\uparrow\rangle_{S}+\beta|\downarrow\rangle_{S}
$$

In the ideal limit, protective measurements of this type maintain the original state and the pointer state is $\theta=$ $\pi|\alpha|^{2}$. But here too, the non-ideal case is the more realistic and we enumerate the possible outcomes [12,13].

- After accounting for the relevant $\frac{1}{T}$ corrections also, the dominant outcome is when the original state is protected and the apparatus outcome is the expectation value of the observable in the original state. But unlike the ideal case, the probability of this happening is no longer unity; instead it happens with probability $P \simeq 1-\frac{c^{2}}{T^{2}}$.

- State collapses to its orthogonal; unlike the protective measurements considered so far, the apparatus state now is uniquely determined to be $\left|d_{\downarrow}\right\rangle_{x}$ ! This happens with probability $P \simeq \frac{1}{T^{2}}$.

- State is protected but apparatus again in $\left|d_{\downarrow}\right\rangle_{x}$, with probability $P \simeq \frac{1}{T^{2}}$.

- It is intriguing that the 'failed' protective measurements now always produce the same apparatus state $\left|d_{\downarrow}\right\rangle_{x}$.

We see that because of non-vanishing probabilities for deviations from the ideal case, perfect ontology is not possible. The last point mentioned above (the failed cases coming with a well defined apparatus state) might give hope that the lack of perfect ontology may somehow be overcome by exploiting this feature. Even though the apparatus state, being fixed, does not convey any information about the initial system state, the state of the system after the measurement being just orthogonal to it, carries all information about it. Unfortunately, no universal unitary transformation can transform an unknown initial state of a qubit to its orthogonal state. But what is worse, there is no way to tell, with only single copies, that the protective measurement has actually failed. The reason is that as long as the pointer states are the ones produced by rotating $\left|d_{\downarrow}\right\rangle$ through $\theta$ around the x-axis, $\left|d_{\downarrow}\right\rangle_{x}$ will have to be expressed as $\frac{1}{\sqrt{2}}\left(\left|d_{\uparrow}\right\rangle+\left|d_{\downarrow}\right\rangle\right.$. This being a superposition of pointer states, there are finite probabilities for different outcomes, and the failed case will behave as in a projective case. This again precludes any perfect ontological significance to these unknown states.

However, protection fails with very low probability. This means protective measurements can give practically full information about a class of unknown states in such a way as to protect the purity of the post-measurement ensemble to a very high degree. Further, a dramatic decrease in the size of the ensemble for state tomography is possible. In other words, protective measurements can provide FAPP ontology to an arbitrary degree, and this can be important and highly useful in this pragmatic sense [13, 14] though from a philosophical point of view they cannot deliver the ontological goods. Because of all these interesting aspects, it is critical that they are subjected to a proper experimental study. For some feasible suggestions, the reader is referred to [13].

\section{Weak Measurements and Ontology}

Now we take up another class of remarkable measurement schemes called Weak Measurements and Weak Value Measurements. These were also discovered by Aharonov and his collaborators [15, 16]. Let us first dispose off the weak value measurements as they are by design unsuited for ontology. These are also called measurements with Post-selection; a post-selection of the system state is made through a projective measurement, following a weak measurement on an initial, possibly unknown state. Obviously, the projective measurements involved in the post-selection stage are invasive on the system. For this reason, this class of measurements cannot have any bearing on the issues of ontology discussed here.

\subsection{Weak Measurements Without Post-Selection}

On the other hand, if no post-selection is made, removing thereby the invasive elements, weak measurements on 
their own appear to be ideal for the ontological issues. As their name suggests, they are minimally invasive, with this degree of invasiveness apparently under full control. Here too, it is possible to make such measurements both on ensembles and on single copies. We consider only the latter here.

As in section 4, let $S$ be the observable of the system with $s_{i},\left|s_{i}\right\rangle_{S}$ its spectrum, which we take to be nondegenerate. The initial states of the system and the apparatus are taken to be pure and as in Equation 23. The measurement interactions are also of the form of Equation 19 discussed in section 5 . But there is an important difference now in that $Q_{A}$ need not be as restrictive as in the Dirac-von Neumann measurement schemes.

The Pointer States of the apparatus denoted by $|p\rangle_{A}$, are taken to be eigenstates of an apparatus observable $P_{A}$. The point of view taken here is that such pointer states form the basis in which the density matrix becomes diagonal as a result of decoherence. They are not always labeled by the mean values of $P_{A}$ in a given state of the apparatus. Therefore, the specification of an apparatus involves some quantum system, along with a decoherence mechanism which picks out the pointer states. The $P_{A}, Q_{A}$ pair need not be canonically conjugate. A detailed account of many important aspects of weak measurements can be found in [17]. In what follows we shall nevertheless stick to the canonical pair for convenience.

The initial apparatus states are taken to be Gaussian states centered around some $p_{0}$. For $p_{0}=0$, we have

$$
\left|\phi_{0}\right\rangle_{A}=N \int d p e^{-\frac{p^{2}}{2 \Delta_{p}^{2}}}|p\rangle_{A}, \quad N^{2} \sqrt{\pi \Delta_{p}^{2}}=1
$$

In projective measurements, the Gaussians are taken to be very narrow that is $\Delta_{p} \ll 1$ so that they approximate pointer states to a high degree. In contrast, for weak measurements, $\Delta_{p} \gg 1$.That means that the initial apparatus state is a very broad superposition of pointer states with practically equal weight for many pointer states. Though even in the weak case, the initial apparatus state is also peaked at $p_{0}=0$, it is not a pointer state. This important point has led to confusing statements in literature.

The measurement interaction is still taken to be impulsive that is the function $g(t)$ is non-vanishing only during a very small duration, say, $-\epsilon<t<\epsilon$. We leave out the details (the reader is referred to [18] for them) and give only the essential results. The post-measurement density matrix turns out to be (in what follows, we shall use the notation $\{\alpha\}$ to indicate the values of the set of all $\alpha$-variables):

$$
\rho_{S A}^{\text {post }}=\int d p|N(p,\{\alpha\})|^{2}|p\rangle\left\langle\left. p\right|_{A} \mid \psi(p,\{\alpha\})\right\rangle\left\langle\left.\psi(p,\{\alpha\})\right|_{S}\right.
$$

where

$$
\begin{gathered}
N(p,\{\alpha\})=N \sqrt{\sum_{i}\left|\alpha_{i}\right|^{2} e^{-\frac{\left(p-s_{i}\right)^{2}}{\Delta_{p}^{2}}}} \\
|\psi(p,\{\alpha\})\rangle=\frac{N}{N(p,\{\alpha\})} \sum_{j} \alpha_{j} e^{-\frac{\left(p-s_{j}\right)^{2}}{2 \Delta_{p}^{2}}}\left|s_{j}\right\rangle_{S}
\end{gathered}
$$

For an ensemble of weak measurements, $P(p,\{\alpha\})=$ $|N(p,\{\alpha\})|^{2}$ being the probability for outcome $p$, the mean outcome is

$$
\langle p\rangle_{\psi}=\int d p p|N(p,\{\alpha\})|^{2}=\sum_{i}\left|\alpha_{i}\right|^{2} s_{i}
$$

yielding the same expectation value as in strong measurements. The variance of the outcomes can be readily calculated to yield

$$
(\Delta p)_{\psi}^{2}=(\Delta p)^{2}+(\Delta S)^{2}
$$

This exposes one of the major weaknesses (!) of weak measurements-the errors in individual measurements are huge. This can be reduced statistically as usual. If one considers averages over $M_{w}$ measurements, the variance in the average, is $\frac{\Delta_{p}}{\sqrt{2 M_{w}}}$. It makes sense to compare different measurement schemes only for a fixed statistical error. Therefore if averaging is done over $M_{s}$ strong measurements,

$$
\frac{\Delta S}{\sqrt{M_{s}}}=\frac{\Delta_{p}}{\sqrt{2 M_{w}}} \rightarrow M_{w}=\left(\frac{\Delta_{p}}{\Delta S}\right)^{2} \frac{M_{s}}{2}
$$

the required resources will be super-massive!

The aspect of weak measurements that has gained great prominence is its alleged non-invasiveness. One possible measure of this non-invasiveness is provided by the postmeasurement reduced density matrix of the system:

$$
\rho_{S}^{\text {post }}=\rho^{\text {ini }}-\frac{1}{4 \Delta_{p}^{2}} \sum_{i, j}\left(s_{i}-s_{j}\right)^{2} \alpha_{i} \alpha_{j}^{*}\left|s_{i}\right\rangle\left\langle s_{j}\right|
$$

Thus, for very large $\Delta_{p}$, the reduced density matrix of the system practically equals that of the initial state.

The combination of an exact estimate for the expectation value, as given by Equation 44, as well as the maintenance of the state to a very high degree as per Equation 47 may give rise to the expectation that weak measurements may offer the best hopes for ontology in quantum mechanics. What would make such an expectation particularly exciting is that these measurements can be done on any state-they appear to offer FAPP ontology for arbitrary states! We investigate this by turning to an analysis of repeated weak measurements on a single copy as given in [18] with particular emphasis on ontology. 
Two aspects that need to be particularly focused upon in this context are (i) how closely the averages of $N$ outcomes approximate the exact expectation values, and, (ii) how the single state gets degraded as a result of multiple weak measurements.

The following schema defines for us repeated weak measurements of the same observable on a single copy [18]: (i) perform a weak measurement of system observable $S$ in state $|\psi\rangle_{S}$ with the apparatus in the state of Equation 40 with very large $\Delta_{p}$, (ii) let the definitive outcome, defined as above, be $p_{1}$, and the single system state be $\left|\psi\left(p_{1},\{\alpha\}\right)\right\rangle_{S}$, (iii) restore the apparatus to its initial state, (iv) repeat step (i), and so on. After $N$ such steps, let the sequence of outcomes be denoted by $p_{1}, p_{2} \ldots, p_{N}$ and the resulting system state by $|\psi(\{p\},\{\alpha\})\rangle_{S}$.

The probability distribution for the first outcome $p_{1}, P^{(1)}\left(p_{1}\right)$ is given by

$$
\left.N^{(1)}\left(p_{1},\{\alpha\}\right)\right|^{2}=\left|N\left(p_{1},\{\alpha\}\right)\right|^{2}
$$

with $N(p,\{\alpha\})$ given by Equation 42 . The corresponding system state is given by $\left|\psi\left(p_{1},\{\alpha\}\right)\right\rangle_{S}$ of Equation 43. Thus the set of $\alpha$ for this state is given by

$$
\alpha_{i}^{(1)}=\frac{N}{N\left(p_{1},\{\alpha\}\right)} e^{-\frac{\left(p_{1}-s_{i}\right)^{2}}{2 \Delta_{p}^{2}}} \alpha_{i}
$$

Since in step (iii) the apparatus state has been restored, the probability distribution $P^{(2)}\left(p_{2}\right)$ for the outcome $p_{2}$ at the end of the second weak measurement, is given by

$$
P^{(2)}\left(p_{2}\right)=\left|N^{(2)}\left(p_{2},\{\alpha\}\right)\right|^{2}=\left|N^{(1)}\left(p_{2},\left\{\alpha^{(1)}\right\}\right)\right|^{2}
$$

Substituting from Equation 49, one gets

$$
P^{(2)}\left(p_{2}\right)=\frac{\left(N^{2}\right)^{2}}{P^{(1)}\left(p_{1}\right)} \sum_{i}\left|\alpha_{i}\right|^{2} \prod_{j=1}^{2} e^{-\frac{\left(p_{j}-s_{i}\right)^{2}}{\Delta_{p}^{2}}}
$$

As stressed in [18], $P^{(2)}\left(p_{2}\right)$ is actually the conditional probability $P\left(p_{2} \mid p_{1}\right)$ of obtaining $p_{2}$ conditional to having already obtained $p_{1}$ (that is the reason for the explicit dependence on $p_{1}$ in Equation 51. The joint probability distribution $P\left(p_{1}, p_{2}\right)$ is therefore given by $P\left(p_{2}, p_{1}\right)=$ $P\left(p_{2} \mid p_{1}\right) P\left(p_{1}\right)$ to give

$$
P\left(p_{1}, p_{2}\right)=\left(N^{2}\right)^{2} \sum_{i}\left|\alpha_{i}\right|^{2} \prod_{j=1}^{2} e^{-\frac{\left(p_{j}-s_{i}\right)^{2}}{\Delta^{2}}}
$$

The state after the second measurement is given by the exact analog of Equation 49.

$$
\alpha_{i}^{(2)}=\frac{N}{N^{(2)}\left(p_{2},\left\{\alpha^{(1)}\right\}\right)} e^{-\frac{\left(p_{2}-s_{i}\right)^{2}}{2 \Delta_{p}^{2}}} \alpha_{i}^{(1)}
$$

It is useful to explicitly write this state:

$$
\mid \psi\left(p_{1}, p_{2},\{\alpha\}\right)=\frac{\sum_{i} \prod_{j=1}^{2} e^{-\frac{\left(p_{j}-s_{i}\right)^{2}}{2 \Delta_{p}^{2}}} \alpha_{i}\left|s_{i}\right\rangle_{S}}{\sqrt{\sum_{i}\left|\alpha_{i}\right|^{2} \prod_{j=1}^{2} e^{-\frac{\left(p_{j}-s_{i}\right)^{2}}{\Delta_{p}^{2}}}}}
$$

It is remarkable that these results are all symmetric in the outcomes $p_{i}$. Equation 52 and Equation 53 readily generalize to the case of $M$ repeated measurements:

$$
\begin{gathered}
P\left(p_{1}, \ldots, p_{M}\right)=\left(N^{2}\right)^{M} \sum_{i}\left|\alpha_{i}\right|^{2} \prod_{j=1}^{M} e^{-\frac{\left(p_{j}-s_{i}\right)^{2}}{\Delta^{2}}} \\
\mid \psi\left(p_{1}, \ldots, p_{M},\{\alpha\}\right)=\frac{\sum_{i} \prod_{j=1}^{M} e^{-\frac{\left(p_{j}-s_{i}\right)^{2}}{2 \Delta_{p}^{2}}} \alpha_{i}\left|s_{i}\right\rangle_{S}}{\sqrt{\sum_{i}\left|\alpha_{i}\right|^{2} \prod_{j=1}^{M} e^{-\frac{\left(p_{j}-s_{i}\right)^{2}}{\Delta_{p}^{2}}}}}
\end{gathered}
$$

\subsection{Consequences for ontology}

The intrinsic randomness of quantum theory makes no aspect of a particular realization predictable. For ensemble measurements the variables are independently distributed and the Central Limit Theorem guarantees that as long as the number of trials is large enough, averages over even particular realizations converge nicely to the true mean. To see what happens in the present context, where the outcomes are clearly not independently distributed, let us study $y_{M}$, the average of $M$ outcomes. The expectation value of $y_{M}$ in the joint probability distribution $P\left(p_{1}, \ldots, p_{M}\right)$ is

$$
\bar{y}_{M}=\frac{1}{M} \int \ldots \int \prod_{i=1}^{M} \sum_{i} p_{i} P(\{p\})=\sum_{i}\left|\alpha_{i}\right|^{2} s_{i}
$$

Which is certainly a remarkable result. With this, the repeated weak measurements on a single copy certainly pass one critical requirement for ontology. The variance in $y_{M}$ can likewise be calculated and it equals $\frac{\Delta_{p}}{\sqrt{2 M}}$. This makes it appear that in principle the errors can be reduced arbitrarily, reminding one of the situation in protective measurements, except that now no restrictions need be placed on the initial states! But such appearances turn out to be highly misleading.

As argued before the crux of the ontology issue lies in the distribution function $P\left(y_{M}\right)$, and not just in its mean and variance. As shown in [18], the distribution function $P\left(y_{M}\right)$ can itself be calculated explicitly. This is in spite 
of the outcomes not being independently distributed. The result is

$$
P\left(y_{M}\right)=\int \ldots \int \prod_{i=1}^{M} d p_{i} P(\{p\}) \delta\left(y_{M}-\frac{\sum_{i} p_{i}}{M}\right)
$$

Using Equation 55, this becomes

$P\left(y_{M}\right)=\sqrt{\frac{M}{\pi \Delta_{p}^{2}}} \sum_{i}\left|\alpha_{i}\right|^{2} e^{-\frac{\left(y_{M}-s_{i}\right)^{2}}{\Delta_{p}^{2}}} \rightarrow \sum_{i}\left|\alpha_{i}\right|^{2} \delta\left(y_{M}-s_{i}\right)$

where we have also displayed the limiting behavior as $M \rightarrow \infty$.

This, as per our discussions earlier, immediately negates not just ontology but even FAPP ontology! In other words, the distribution of $y_{M}$ is not only not peaked at the true average, with errors decreasing as $M^{-1 / 2}$, it is actually a weighted sum of sharp distributions peaked around the eigenvalues, exactly as in the strong measurement case. This means that averages over outcomes of a particular realization will be eigenvalues, occurring randomly but with probability $\left|\alpha_{i}\right|^{2}$. It then follows that averages over outcomes of a particular realization do not give any information about the initial state, precisely as in the case of the invasive strong measurements where there can clearly be no ontology! Ensemble measurements again become inevitable.

The other issue to be settled in this context is whether the repeated measurements on single copies are invasive or not. It turns out that a very large number of repeated weak measurements on a single copy has the same invasive effect as a strong measurement. This can be seen by examining the expectation value of the system reduced density matrix, $\rho_{>}^{\text {rep }}$ :

$$
\rho_{>}^{\text {rep }}=\rho-\sum_{i, j} \alpha_{i} \alpha_{j}^{*}\left(1-e^{-\frac{M\left(s_{i}-s_{j}\right)^{2}}{4 \Delta_{p}^{2}}}\right)\left|s_{i}\right\rangle\left\langle s_{j}\right|
$$

It is seen that as $M$ gets larger and larger, there is significant change in the system state. In the limit $M \rightarrow \infty$, the off-diagonal parts of the density matrix get completely quenched, as in decoherence, and the density matrix takes the diagonal form in the eigenstate of $S$ basis:

$$
\rho_{>}^{\text {rep }} \rightarrow \sum_{i}\left|\alpha_{i}\right|^{2}\left|s_{i}\right\rangle\left\langle s_{i}\right|
$$

which is exactly the post-measurement density matrix in the case of a strong measurement! The sequence of system states of Equation 56 is a random walk on the state space of the system (see also [19]). It follows from Equation 43 that the eigenstates of $S$ are the fixed points of the probabilistic map that generates this walk. Presumably each walk terminates in one of the eigenstates but which eigenstate it terminates in is unpredictable.

\subsection{Other equivalent results}

Alter and Yamamoto have obtained a number of very significant results about the possibility of obtaining information about single quantum systems [3, 20, 21]. In particular they also gave an analysis based on joint and conditional probabilities applied to repeated weak quantum non-demolition measurements on a single state [21]. They too obtained evolutions resembling random walks in state space. They concluded that it is not possible to obtain any information on unknown single states from the statistics of repeated measurements. The degradation of the state and relation to projective measurements were not explicitly studied. In another work, they found connections between Quantum Zeno Effect and the problem of repeated measurements and again concluded that it is impossible to determine the quantum state of a single system. Our results on information cloning and the general results from optimal cloning discussed in the next two sections show that it may be possible to obtain partial results.

In a very interesting approach to these ontological questions, Paraoanu has investigated these issues within what he calls partial measurements [22,23]. By employing a combination of repeated such measurements on a single state and the possibility of reversing such measurements, he too has concluded the impossibility of obtaining any information about single unknown states. The invasive aspects as well as the connections to strong measurements are not explored here either.

\section{Information Cloning and Ontology}

As we saw in subsection 3.2, a subtle inner consistency of quantum theory prevents determining the unknown state of a single copy by trying to make many clones of it. We had, however, proposed what we called information cloning in [24]. The main idea was to make many copies of an unknown state which are however not identical to the original state, but contain the same amount of information as the original. Now we discuss the implications for ontology of such a cloning scheme [25].

The details of how this type of cloning can be used to determine the state of a single unknown coherent state of quantum harmonic oscillators can be found in [25]. In the case of coherent states of harmonic oscillators (say, in one dimension), complete information about the state is contained in a single complex coherency parameter $\alpha$. Thus by information cloning what we mean is the ability to make arbitrary number of copies of coherent states whose coherency parameter is $c(N) \alpha$ where $\alpha$ is 
the coherency parameter of the unknown coherent state and $c(N)$ is a known constant depending on the number of copies made.

To this end consider $1+N$ systems of harmonic oscillators whose creation and annihilation operators are the set $\left(a, a^{\dagger}\right),\left(b_{k}, b_{k}^{\dagger}\right)$ (where the index $k$ takes on values $\left.1, \ldots, N\right)$. The $a$ oscillators represent the original unknown state, and the $b$ oscillators represent the information clones. These operators satisfy the commutation relations

$$
\begin{aligned}
{\left[a, a^{\dagger}\right] } & =1 \\
{\left[b_{j}, b_{k}^{\dagger}\right] } & =\delta_{j k} \\
{\left[a, b_{k}\right] } & =0 \\
{\left[a^{\dagger}, b_{k}\right] } & =0
\end{aligned}
$$

Coherent states parametrized by the complex number $\alpha$ are given by

$$
|\alpha\rangle=D(\alpha)|0\rangle
$$

where $|0\rangle$ is the ground state and the unitary operator $D(\alpha)$ is given by

$$
D(\alpha)=e^{\alpha a^{\dagger}-\alpha^{*} a}
$$

We view the information cloning to be a unitary process. The initial composite state can be taken to be a disentangled state containing the unknown initial coherent state and some known states of the $b$-oscillators. It turns out to be best to take them also to be coherent states. In other words, the state before information cloning is taken as $|\alpha\rangle\left|\beta_{1}\right\rangle_{1}\left|\beta_{2}\right\rangle_{2} \ldots\left|\beta_{N}\right\rangle_{N}$, where $\alpha$ is unknown while $\beta_{i}$ are known to very high accuracy. Consider the action of the unitary transformation

$$
U=e^{t\left(a^{\dagger} \otimes \sum_{j} r_{j} b_{j}-a \otimes \sum_{j} r_{j} b_{j}^{\dagger}\right)}
$$

The most general unitary transformation of this type would involve complex $r_{j}$ 's. But this can be reduced to the present form through suitable redefinitions of the phases of the creation and annihilation operators [24]. Of course, such redefinitions should maintain the algebra of Equation 62 . The process implemented by this unitary transformation is well known in optics and is called the beam splitter. But it is very important to appreciate that what we are dealing with here is when this acts on a single photon state, a circumstance in which the notion of a beam is neither meaningful nor useful. By an application of the Baker-Campbell-Hausdorff identity and the fact that $U|0\rangle|0\rangle_{1} . .|0\rangle_{N}=|0\rangle|0\rangle_{1} . .|0\rangle_{N}$ it follows that the resulting state is also a disentangled set of coherent states expressed by

$$
\left|\alpha^{\prime}\right\rangle\left|\beta_{1}^{\prime}\right\rangle_{1} . .\left|\beta_{N}^{\prime}\right\rangle_{N}=U|\alpha\rangle\left|\beta_{1}\right\rangle_{1} . .\left|\beta_{N}\right\rangle_{N}
$$

In other words, the unitary transformation $U$ acting on various coherent states induces another unitary transformation $\mathcal{U}$ among the coherency parameters. Details can be found in [24]; we merely give the final result and discuss its physical implications. Let us define

$$
\begin{array}{r}
a(t)=U a U^{\dagger} \\
b_{j}(t)=U b_{j} U^{\dagger}
\end{array}
$$

The explicit form of the transformation induced on the parameters $\left(\alpha, \beta_{j}\right)$ can be represented by the matrix $\mathcal{U}$ as

$$
\alpha_{a}(t)=\mathcal{U}_{a b} \alpha_{b} .
$$

where we have introduced the notation $\alpha_{a}$ with $a=$ $1, \ldots, N+1$ such that

$$
\begin{aligned}
& \alpha_{1}=\alpha \\
& \alpha_{k}=\beta_{k-1} k \geq 2
\end{aligned}
$$

Then we have

$$
\mathcal{U}_{1 a}=\left(\begin{array}{lllll}
\cos R t & \frac{r_{1}}{R} \sin R t & . . & . . & \frac{r_{N}}{R} \sin R t
\end{array}\right)
$$

where $R=\sqrt{\sum_{j} r_{j}^{2}}$ and

$$
\mathcal{U}_{a b}=-\frac{r_{a-1}}{R} \sin R t \delta_{b 1}+\left(1-\delta_{b 1}\right) M_{a-1, b-1}
$$

where Equation 75 is defined for $a \geq 2$. Equivalently

$$
\mathcal{U}=\left(\begin{array}{ccccc}
\cos R t & \frac{r_{1}}{R} \sin R t & . . & . . & \frac{r_{N}}{R} \sin R t \\
-\frac{r_{1}}{R} \sin R t & M_{11} & . . & . . & M_{1 N} \\
. . & . . & . . & . . & \\
. . & . . & . . & . . & \\
-\frac{r_{N}}{R} \sin R t & M_{N 1} & . . & . . & M_{N N}
\end{array}\right)
$$

It is best to choose $\left\{\beta_{i}, r_{i}\right\}$ in such a way that all $\beta_{i}(t)$ become identical and we get $N$ identical copies. This happens only when $r_{i}=r, \beta_{i}=\beta$. In that case we have

$$
\beta_{i}(t)=-\frac{\alpha}{\sqrt{N}} \sin R t+\beta \cos R t
$$

There is still the freedom to choose Rt. Let us first consider the choice of $\sin R t=-1$ which gives $N$ copies of the state $\left|\frac{\alpha}{\sqrt{N}}\right\rangle$. This is what we called information cloning in $[24]$ as the states $\left|\frac{\alpha}{\sqrt{N}}\right\rangle$ and $|\alpha\rangle$ have the same information content. This particular choice of $R t$ will be seen to be optimal in the sense that it gives the least variance in the estimation of $\alpha$. In this case the value of $\beta$ is immaterial.

It is easily seen that Equation 7 does not pose any difficulties for information cloning, as it did for universal cloning! Now we can address the ontology issue by attempting to use the $N$-information clones for an ensemble determination of the information-clone state first, and then a state determination of the original unknown state subsequently, by using the fact that the information clone 
has the same information content as the original. More specifically, we can use the $N$ copies of $\left|\frac{\alpha}{\sqrt{N}}\right\rangle$ to make ensemble measurements to estimate $\frac{\alpha}{\sqrt{N}}$ and $\alpha$.

One can already sense some limitations of the method: usually, the statistical errors can be made arbitrarily small by making the ensemble size larger and larger. However, in our proposal even though the number of copies $N$ can be made arbitrarily large, at least in principle, the coherency parameter given by $\frac{\alpha}{\sqrt{N}}$ becomes arbitrarily small while the uncertainties in $\alpha$, being characteristic of coherent states, remain the same as in the original state. We now address the question as to how best the original state can be reconstructed.

On introducing the Hermitian momentum and position operators $\hat{p}, \hat{x}$ through

$$
\begin{aligned}
& \hat{x}=\frac{\left(a+a^{\dagger}\right)}{\sqrt{2}} \\
& \hat{p}=\frac{\left(a-a^{\dagger}\right)}{\sqrt{2} \imath}
\end{aligned}
$$

the probability distributions for position and momentum in the coherent state $\left|\frac{\alpha}{\sqrt{N}}\right\rangle$ are given by

$$
\begin{aligned}
\left|\psi_{\text {clone }}(x)\right|^{2} & =\frac{1}{\sqrt{\pi}} e^{-\left(x-\sqrt{\frac{2}{N}} \alpha_{R}\right)^{2}} \\
\left|\psi_{\text {clone }}(p)\right|^{2} & =\frac{1}{\sqrt{\pi}} e^{-\left(p-\sqrt{\frac{2}{N}} \alpha_{I}\right)^{2}}
\end{aligned}
$$

Let us distribute our $N$-copies into two groups of $\frac{N}{2}$ each and use one to estimate $\alpha_{R}$ through position measurements and the other to estimate $\alpha_{I}$ through momentum measurements. Let $y_{N}$ denote the average value of the position obtained in $\frac{N}{2}$ measurements and let $z_{N}$ denote the average value of momentum also obtained in $\frac{N}{2}$ measurements. The central limit theorem states that the probability distributions for $y_{N}, z_{N}$ are given by

$$
\begin{aligned}
& f_{x}\left(y_{N}\right)=\sqrt{\frac{N}{2 \pi}} e^{-\frac{N}{2}\left(y_{N}-\sqrt{\frac{2}{N}} \alpha_{R}\right)^{2}} \\
& f_{p}\left(z_{N}\right)=\sqrt{\frac{N}{2 \pi}} e^{-\frac{N}{2}\left(z_{N}-\sqrt{\frac{2}{N}} \alpha_{I}\right)^{2}}
\end{aligned}
$$

It is more instructive to recast these as the probability distributions for $\bar{\alpha}_{R, N}, \bar{\alpha}_{I, N}$, the average over $N$ measurements of $\alpha_{R}, \alpha_{I}$ :

$$
\begin{aligned}
f_{R}\left(\bar{\alpha}_{R, N}\right) & =\frac{1}{\sqrt{\pi}} e^{-\left(\bar{\alpha}_{R, N}-\alpha_{R}\right)^{2}} \\
f_{I}\left(\bar{\alpha}_{I, N}\right) & =\frac{1}{\sqrt{\pi}} e^{-\left(\bar{\alpha}_{I, N}-\alpha_{I}\right)^{2}}
\end{aligned}
$$

Thus the original unknown $\alpha$ is correctly estimated, in the sense that the above distributions peak precisely at the coherency parameter $\alpha$ of the original state. But this is not enough and one needs to know the reliability of this estimate. For that one needs the variances. The variances for $\alpha_{N}$ are easily found out from Equation 82 .

$$
\Delta \alpha_{R, N}=\Delta \alpha_{I, N}=\frac{1}{\sqrt{2}}
$$

Thus, while the statistical error in usual measurements goes as $\frac{1}{\sqrt{N}}$, and can be made arbitrarily small by making $N$ large enough, information cloning gives an error that is fixed and equal to the variance associated with the original unknown state. For coherent states with large enough $\alpha$, even these errors are quite reasonable. Another figure of merit, the so called Fidelity has also been adopted in [24, 26-30]. That fidelity for information cloning works out to $\frac{1}{2}$ [24], the maximum possible for Gaussian Cloning [26-33]. Therefore, fidelity on its own may give an unnecessarily pessimistic picture. Comparison between information cloning and optimal cloning mentioned above will again be made in section 8 .

Thus we have shown that even when the coherent state is unknown single state, information cloning will allow its determination, but with fixed statistical errors. Nevertheless, it is a great improvement from not being able to know anything at all about the unknown state.

A comparison with our technical criteria for ontology reveals that again there is no perfect ontology but indeed there is FAPP ontology of the first kind. In contrast, protective measurements gave a FAPP ontology of the second kind. In the protective case the FAPP ontology could approach perfect ontology arbitrarily close, but never equal it. In both cases, one had to restrict the classes of states for which they would work and the restricted class did not allow linear superpositions.

\section{Approximate Cloning and Ontology}

Though the No-cloning theorem forbids making perfect clones of an unknown state, there seems nothing against making imperfect copies. The information cloning of the previous section was a particularly interesting variant of this theme. Then the obvious question is the closeness to perfect cloning that can be achieved. There has been an explosion of interest in this question and the reader is referred to [31] for a comprehensive review. We shall only examine the so called optimal cloning [26-30, 32, 35] (see [31] for a review), from the ontological point of view. The details are not that critical to understanding the broad implications and chief conclusions.

In these implementations, one starts with the original unknown state $|\alpha\rangle$ belonging to the Hilbert space $\mathcal{H}_{A}$, a 
number of ancillary states, also known as blank states, $\left|b_{0}\right\rangle,\left|b_{1}\right\rangle \ldots\left|b_{N}\right\rangle$. The ancillaries are known states. This is the general setup for all cloning processes. The ancillaries belong to the Hilbert spaces $\mathcal{H}_{B_{i}}$; each of them is isomorphic to $\mathcal{H}_{A}$. Unlike the information cloning case, a number of additional states called machine states, also known, $\left|m_{0}\right\rangle,\left|m_{1}\right\rangle \ldots . .\left|m_{M}\right\rangle$ all belonging to the Hilbert spaces isomorphic to, say, $\mathcal{H}_{M}$, are also considered. The combined Hilbert space has the structure $\mathcal{H}_{A} \otimes \mathcal{H}_{M} \otimes \prod_{i} \mathcal{H}_{B_{i}}$.

A general cloning transformation $\mathcal{T}$ has the effect

$$
|\alpha\rangle \prod_{0}^{N}\left|b_{i}\right\rangle \prod_{0}^{M}\left|m_{j}\right\rangle \stackrel{\mathcal{T}}{\rightarrow} \sum_{i, j, k} d_{i j k}\left|a_{i}\right\rangle \prod_{j}^{M}\left|\beta_{j}\right\rangle \prod_{k}^{N}\left|\gamma_{k}\right\rangle
$$

Such a general cloning is said to be optimal if it satisfies the two conditions: (i) all the reduced density matrices $\rho_{i_{0}}$ obtained by tracing over the $\mathcal{H}_{A}$ states, the machine states and all the blank states except those belonging to $\mathcal{H}_{B_{i_{0}}}$, are all identical and (ii) each of them has maximum overlap with the original unknown state $|\alpha\rangle$ that is with the maximum possible value of $\left\langle\alpha\left|\rho_{i_{0}}\right| \alpha\right\rangle$. The reduced density matrices are in general mixed.

In the case of information cloning, the clones were all disentangled and one could use all of them at a time for carrying out measurements of one's choice. In the case of optimal cloning, in general the clones could be in entangled states. Depending on such details, it could even be that that at any given time it is possible to realize only a few of the reduced matrices $\rho_{i}$ as different values of $i$ require tracing over different states.

As can be gathered from [31] and the many references there, there are various types of optimal clonings. But for the ontological questions, only a part of them are of interest. Firstly, we need only look at the so called universal types as these can produce clones of unknown states. The so called state dependent cloning is not of interest. The information cloning that we discussed earlier is state dependent in one way as it can work only with coherent states, but it is also somewhat universal in the sense that the input state can be any coherent state. In fact, it is a particular case of Gaussian cloning [26-33]. Secondly, even among the universal optimal cloning there are results for the so called $N \rightarrow M$ type clonings. Here $N$ is the number of copies of the unknown initial state (usually pure) and $M$ the number of clones (usually mixed). For our ontological considerations, only $1 \rightarrow M$ types are relevant.

Let us first consider the case where the input Hilbert space is finite dimensional, and specifically consider only cubits. We shall only look at a few illustrative aspects. For qubits, the fidelity $F$, which is the overlap of the clone with the original, is, given by

$$
F(N, M)=\frac{M N+M+N}{M(N+2)}
$$

for the $N \rightarrow M$ case. The clone state is of the form (with $\operatorname{tr} \rho \cdot \rho^{\perp}=0$ ),

$$
\rho^{\text {clone }}=F \rho^{\text {ini }}+(1-F) \rho^{\perp}
$$

The accuracy of the state determination with the clones requires as large a $M$ as possible. Therefore, for $N=1$ and $M \rightarrow \infty$, one has $F=\frac{2}{3}$. In fact, for an arbitrary $M$,

$$
F(1, M)=\frac{2 M+1}{3 M}
$$

The largest value, for the non-trivial case $1 \rightarrow 2$ is $\frac{5}{6}$. But with only two clones the errors in the state determination are very high. But as $M$ is increased, to get more accurate state determination, $F$ decreases, reaching the limiting value of $\frac{2}{3}$. In that case though the errors are very small, the estimates for expectation values of observables deviates significantly from the true values. For example, for observables $O$ with zero expectation values in $\rho^{\perp}$, one finds

$$
\langle O\rangle_{\text {clone }}=\frac{2}{3}\langle O\rangle_{\text {true }}
$$

failing even the first criterion for ontology rather poorly. Unlike the information cloning case, where the error was independent of the input state, here the error is a finite fraction $\left(\frac{1}{3}\right)$ of the expectation value. The resources required even to reach this are impractically large $(M \rightarrow \infty)$.

Of course, it is inappropriate to use the results obtained for optimal cloning of qubits to make a comparison with information cloning which is really a case of infinitedimensional Hilbert space. But results are also available for optimal cloning for arbitrary, but finite dimensional Hilbert space. As an intermediary to considering continuous variable cloning, let us consider Werner's results [33] for $d$-dimensional Hilbert spaces. The formula for the fidelity of $N \rightarrow M$ cloning is

$$
F(N, M)=\frac{N}{M}+\frac{(M-N)(N+1)}{M(N+d)}
$$

The clone state is given by

$$
\rho^{\text {clone }}=\eta(N, M) \rho^{\text {ini }}+(1-\eta(N, M)) \frac{I}{d}
$$

where

$$
\eta(N, M)=\frac{N}{M} \frac{M+d}{N+d}
$$


Let us look at the continuous case by letting $d \rightarrow \infty$ first. While the fidelity approaches the limit $\frac{N}{M}$, the density matrix formula is much more tricky. Now if apply this formula for fidelity to $N=1, M \rightarrow \infty$ limit relevant for our ontological concerns, we see that the fidelity vanishes!

This is because of the attempt to find a universal cloner for continuous variable case. Let us lower the expectations and consider only coherent states. It has been shown that the fidelity is bounded by

$$
F(N, M) \leq \frac{M N}{M N+M-N}
$$

The clone state is a mixture of coherent states centered around the unknown initial coherent state. Its explicit form is given by (see eqn.(53) of [31], but watch for a typo!):

$$
\rho^{\text {clone }}(\alpha)=\frac{1}{\pi \sigma(N, M)^{2}} \int d^{2} \beta e^{-\frac{|\beta|^{2}}{\sigma(N, M)^{2}}}|\alpha+\beta\rangle\langle\alpha+\beta|
$$

where $\sigma(N, M)$ stands for

$$
\sigma(N, M)^{2}=\frac{1}{N}-\frac{1}{M} \geq 0
$$

Returning to the ontology issue, we set $N=1$. It is easy to verify that the mean values of $x$ and $p$ in the clone state of Equation 93 are exactly the same as in the unknown original coherent state. This was so in the case of information cloning too. But the variances in $x$ and $p$ for the clone state turn out to be

$$
(\Delta x)_{\text {clone }}^{2}=\frac{1}{2}+\sigma(1, M)^{2}=\left(\Delta_{p}\right)_{\text {clone }}^{2}
$$

Like the information cloning case, these variances are the same for all coherent states. But irrespective of $M$, the variances are worse here than there. Again there is only FAPP ontology, of a somewhat worse quality.

\subsection{Probabilistic Cloning}

What was described till now can be called deterministic cloning. There are also probabilistic cloning machines. The reader is referred to [36] to get an understanding of these. Many features and implementations are different and these cloning devices are very interesting. But from our ontological perspective, the situation is not too different; again the mean values can approach the true values and the errors cannot be completely eliminated. One can ascribe a FAPP ontology with figures of merit determined by both of these.

\section{Conclusions}

In this paper we have carefully examined the issue of obtaining information about the state of a single quantum system. We have equated the ability to obtain such information with the concept of ontology in quantum mechanics. We have given a precise technical characterization of this concept and examined the implications of a large variety of quantum measurements including projective measurements, protective measurements, weak measurements (including weak quantum non-demolition measurements) and the so called partial measurements. We have also examined the issue in the light of the nocloning theorem on the one hand, and in the light of a variety of cloning techniques.

The impossibility of gaining information about a single quantum state is considered to be the basic tenet of quantum mechanics. Admittedly, it was based on the picture of quantum measurements that dominated during the early development of quantum theory. Central to that line of thinking were the highly invasive nature of the eigenvalue-eigenstate based projective measurements. In view of the highly invasive nature of such measurements, that thinking seemed almost obvious. But what is surprising now is that when even novel measurement schemes like weak measurements, partial measurements are around, which make such a tenet far from obvious, it still remains rock solid. Now the results that even these seemingly non-invasive measurement schemes simply cannot coax any information out of generic single states make this lack of ontology deep and perplexing, as if they are the foundational principles of quantum theory. Nevertheless, that schemes like protective measurements, information cloning in particular and optimal cloning in general exist to provide a silver lining in the form of what we have called FAPP ontology is also equally perplexing. What general principles are lurking behind these is something that all those trying to fathom the depths of quantum theory will be eagerly searching for.

\section{Acknowledgments}

I thank Tata Institute of Fundamental Research Centre for Interdisciplinary Sciences, Hyderabad for its warm hospitality during which this work was completed. I am thankful to the late Jeeva Anandan for many discussions close to the theme of this article and Tabish Qureshi for raising very interesting questions about completely invasive measurements which could reveal unknown states, and about possible implications of teleportation for ontology. I acknowledge support from the Department of Science and Technology to the project IR/S2/PU-001/2008. 


\section{References}

[1] Dirac PAM. The Principles of Quantum Mechanics, 1st edition. Oxford: Oxford University Press, 1930.

[2] Dirac PAM. The Principles of Quantum Mechanics, 2nd edition. Oxford: Oxford University Press, 1934.

[3] Alter O, Yamamoto Y. Quantum Measurement of a Single System. New York: Wiley, 2001.

[4] Wiseman HM, Milburn GJ. Quantum Measurement and Control. Cambridge: Cambridge University Press, 2010.

[5] Hari Dass ND. The superposition principle in quantum mechanics-did the rock enter the foundation surreptitiously? http://arxiv.org/abs/1311. 4275

[6] Wootters WK, Zurek WH. A single quantum cannot be cloned. Nature 1982; 299 (5886): 802-803. http://dx.doi.org/10.1038/299802a0

[7] Dieks D. Communication by EPR devices. Physics Letters A 1982; 92 (6): 271-272.http://dx.doi. org/10.1016/0375-9601(82)90084-6

[8] Aharonov Y, Vaidman L. Measurement of the Schrödinger wave of a single particle. Physics Letters A 1993; 178 (1-2): 38-42. http://dx. doi.org/10.1016/0375-9601(93)90724-E http://arxiv.org/abs/hep-th/9304147

[9] Aharonov Y, Anandan J, Vaidman L. Meaning of the wave function. Physical Review A 1993; 47 (6): 4616-4626. http://dx.doi.org/10.1103/ PhysRevA.47.4616

[10] Hari Dass ND, Qureshi T. Critique of protective measurements. Physical Review A 1999; 59 (4): 2590-2601.http://dx.doi.org/10.1103/ PhysRevA.59.2590

[11] Alter O, Yamamoto Y. Protective measurement of the wave function of a single squeezed harmonicoscillator state. Physical Review A 1996; 53 (5): R2911-R2914. http://dx.doi.org/10.1103/ PhysRevA.53.R2911

[12] Das A, Hari Dass ND. An alternate model for protective measurements of two-level systems. http: //arxiv.org/abs/quant-ph/0410098

[13] Hari Dass ND. Experiments for realising pragmatic protective measurements. AIP Conference Proceedings 2011; 1384 (1): 51-58.http://dx.doi.org/ $10.1063 / 1.3635843$
[14] Hari Dass ND. Cold atoms for testing quantum mechanics and parity violation in gravitation. http: //arxiv.org/abs/quant-ph/9908085

[15] Aharonov Y, Albert DZ, Vaidman L. How the result of a measurement of a component of the spin of a spin-1/2 particle can turn out to be 100 . Physical Review Letters 1988; 60 (14): 1351-1354.http:// dx.doi.org/10.1103/PhysRevLett.60.1351

[16] Aharonov Y, Vaidman L. Properties of a quantum system during the time interval between two measurements. Physical Review A 1990; 41 (1): 11-20. http://dx.doi.org/10.1103/ PhysRevA.41.11

[17] Kofman AG, Ashhab S, Nori F. Nonperturbative theory of weak pre- and postselected measurements. Physics Reports 2012; 520 (2): 43-133. http://dx.doi. org $/ 10.1016 / j$.physrep.2012.07.001 http://arxiv.org/abs/1109.6315

[18] Hari Dass ND. Repeated weak measurements on a single copy are invasive. http://arxiv.org/ abs/1406.0270

[19] Korotkov AN. Continuous quantum measurement of a double dot. Physical Review B 1999; 60 (8): 5737-5742.http://dx.doi.org/10.1103/ PhysRevB.60.5737

[20] Alter O, Yamamoto Y. Quantum Zeno effect and the impossibility of determining the quantum state of a single system. Physical Review A 1997; 55 (4): R2499-R2502. http://dx.doi.org/10. 1103/PhysRevA. 55.R2499

[21] Alter O, Yamamoto Y. Inhibition of the measurement of the wave function of a single quantum system in repeated weak quantum nondemolition measurements. Physical Review Letters 1995; 74 (21): 4106-4109. http://dx.doi.org/10. 1103/PhysRevLett.74.4106

[22] Paraoanu GS. Partial measurements and the realization of quantum-mechanical counterfactuals. Foundations of Physics 2011; 41 (7): 1214-1235. http: //dx.doi.org/10.1007/s10701-011-9542-7 http://arxiv.org/abs/1105.2021

[23] Paraoanu GS. Extraction of information from a single quantum. Physical Review A 2011; 83 (4): 044101. http://dx.doi.org/10.1103/ PhysRevA.83.044101 
[24] Hari Dass ND, Ganesh P. Information cloning of harmonic oscillator coherent states. Pramana - Journal of Physics 2002; 59 (2): 263-267. http://dx. doi.org/10.1007/s12043-002-0116-2 http: //arxiv.org/abs/quant-ph/0202020

[25] Hari Dass ND. Unknown single oscillator coherent states do have statistical significance. http: //arxiv.org/abs/1005.4486

[26] Cerf NJ, Ipe A, Rottenberg X. Cloning of continuous quantum variables. Physical Review Letters 2000; 85 (8): 1754-1757. http://dx.doi.org/ 10.1103/PhysRevLett. 85.1754

[27] Cerf NJ, Iblisdir S. Optimal $N$-to- $M$ cloning of conjugate quantum variables. Physical Review A 2000; 62 (4): 040301.http://dx.doi.org/10.1103/ PhysRevA.62.040301

[28] Braunstein SL, Cerf NJ, Iblisdir S, van Loock P, Massar S. Optimal cloning of coherent states with a linear amplifier and beam splitters. Physical Review Letters 2001; 86 (21): 4938-4941. http:// dx.doi.org/10.1103/PhysRevLett.86.4938

[29] Bruß D, Macchiavello C. Optimal state estimation for $d$-dimensional quantum systems. Physics Letters A 1999; 253 (5-6): 249-251.http://dx.doi. org/10.1016/S0375-9601(99)00099-7 http: //arxiv.org/abs/quant-ph/9812016

[30] Acín A, Latorre JI, Pascual P. Optimal generalized quantum measurements for arbitrary spin systems. Physical Review A 2000; 61 (2): 022113. http:// dx.doi.org/10.1103/PhysRevA.61.022113
[31] Scarani V, Iblisdir S, Gisin N, Acín A. Quantum cloning. Reviews of Modern Physics 2005; 77 (4): 1225-1256.http://dx.doi.org/10.1103/ RevModPhys.77.1225

[32] Lindblad G. Cloning the quantum oscillator. Journal of Physics A: Mathematical and General 2000; 33 (28): 5059-5076. http://dx.doi.org/10. 1088/0305-4470/33/28/310

[33] Keyl M, Werner RF. Optimal cloning of pure states, testing single clones. Journal of Mathematical Physics 1999; 40 (7): 3283-3299. http://dx.doi.org/10.1063/1.532887 http://arxiv.org/abs/quant-ph/9807010

[34] Fiurášek J. Optical implementation of continuousvariable quantum cloning machines. Physical Review Letters 2001; 86 (21): 4942-4945. http:// dx.doi.org/10.1103/PhysRevLett.86.4942

[35] Grosshans F, Grangier P. Quantum cloning and teleportation criteria for continuous quantum variables. Physical Review A 2001; 64 (1): 010301. http:// dx.doi.org/10.1103/PhysRevA.64.010301 http://arxiv.org/abs/quant-ph/0012121

[36] Duan L-M, Guo G-C. Probabilistic cloning and identification of linearly independent quantum states. Physical Review Letters 1998; 80 (22): 4999-5002. http://dx doi.org/10.1103/PhysRevLett. 80.4999 http://arxiv.org/abs/quant-ph/9804064 